\title{
Operations research at CLAIO XVII (ALIO/SMIO Joint International Meeting)
}

\author{
Roger Z. Ríos-Mercado ${ }^{1}$. \\ José Luis González-Velarde ${ }^{2}$ • Manuel Laguna ${ }^{3}$
}

Published online: 8 November 2017

(C) Springer Science+Business Media, LLC 2017

\section{CLAIO history}

The Association of Latin-Iberoamerican Operational Research Societies (ALIO) has been holding its biannual conference (CLAIO), sponsored by ALIO and the different Operations Research (OR) societies of the organizing countries, since 1982. These conferences have succeeded in achieving the goals of fostering the exchange of research among leading investigators, establishing and deepening ties between researchers and practitioners, and helping graduate and undergraduate students in their early professional development. Traditionally, the CLAIO series of conferences have highlighted recent developments in theory, models, algorithms, and applications of operations research in the broadest sense. These contributions have not been limited to ALIO researchers, as we have seen how researchers from the rest of the world have been participating very actively in the past few years.

From its first very first meeting, the CLAIO conference has traveled through different cities including Rio de Janeiro (1982, 1988, 1996, 2012), Buenos Aires (1984, 1990, 1998, 2010), Santiago de Chile (1986, 1994, 2016), Mexico City (1992, 2000), Concepción, Chile (2002), Havana (2004), Montevideo (2006), Cartagena de Indias (2008), and Monterrey, Mexico (2014).

Roger Z. Ríos-Mercado roger@yalma.fime.uanl.mx

José Luis González-Velarde gonzalez.velarde@itesm.mx

Manuel Laguna laguna@colorado.edu

1 Universidad Autónoma de Nuevo León, Systems Engineering Program, AP 111-F, Cd. Universitaria, San Nicolás de los Garza, NL 66450, Mexico

2 Center for Quality and Manufacturing, Tecnológico de Monterrey, Ave. Eugenio Garza Sada 2501 Sur, Col. Tecnológico, Monterrey, NL 64849, Mexico

3 Leeds School of Business, University of Colorado, 419 UCB, Boulder, CO 80309, USA 


\section{The partnership with the Annals of Operations Research}

The partnership between ALIO and the Annals of Operations Research (ANOR) began with CLAIO X, held in Mexico City (2000). The late Peter Hammer, the ANOR Editor-in-Chief at that time, embraced and supported this project from the outset. After Professor Hammer passed away, Professor Boros, took over as the journal EIC, and has continued to support these CLAIO special issues. This collaboration has paid off through the years as we have seen collections of very high-quality papers published in the special issues of the Annals of Operations Research devoted to CLAIO. Volumes 116 and 117 of ANOR were dedicated to CLAIO X (Mexico City), and Volumes 138, 157, 169, and 199 to CLAIO XI (Concepción, Chile), CLAIO XII (Havana), CLAIO XIII (Montevideo), and CLAIO XV (Buenos Aires), respectively.

\section{About CLAIO XVII}

In particular, the present volume contains a selection of high quality papers presented at the ALIO/SMIO Joint International Meeting, held in Monterrey, Mexico, October 2014. In addition, the call for papers was open to the OR community worldwide, so we also received a few submissions not presented at CLAIO. The Meeting was jointly organized by ALIO and the Mexican Operations Research Society (SMIO), corresponding to CLAIO XVII and the 3rd SMIO Annual Conference (CSMIO III). Additional funding was provided by the Mexican Council for Science and Technology (CONACyT). Further details about the organization, statistics, and social activities of the conference can be found in González-Velarde and RíosMercado (2014). Among the more than 400 abstracts presented at the conference, 96 full papers were received by the special issue guest editors. Each of these went through a peerreviewing process under the journal's strict criteria for scientific merit. At the end of the process, 29 manuscripts were accepted and are published in this volume. The following section presents an overview of the contents of this issue.

\section{Research contributions}

In the present volume, the contributions have been organized into the following areas: "Simulation and Queueing Models," "Inventory Management," "Supply Chains and Logistics," "Scheduling and Project Management," "Forest Planning," "Integer and Semi-infinite Programming," "Location Science," "Cutting and Packing," and "Metaheuristics."

\subsection{Simulation and queueing models}

In their paper "Impact on yard efficiency of a truck appointment system for a port terminal," Ramírez Nafarrate, González-Ramírez, Smith, Guerra-Olivares, and Voß, analyze potential configurations of a Truck Appointment System (TAS) in a maritime port terminal and evaluate the impacts that implementing this type of system may have on yard operations, particularly in the reduction of container re-handles and truck turnaround times. They propose a discreteevent simulation model and assess it using historical data from the Port of Arica, Chile. This is followed by the paper "An agent-based simulation approach to congestion management for the Colombian electricity market" by Zambrano and Olaya, who present a hybrid optimization and agent based simulation approach for analyzing changes in transmission pricing 
in Colombia. The model has a module for transmission based on optimal flow problems and a market module that simulates the market operation. They show the usefulness of their proposed approach by evaluating the performance of generation-transmission expansion plans. Song and Zhuang's paper, "Two-stage security screening strategies in the face of strategic applicants, congestions and screening errors," analyzes the optimal screening policies in an imperfect two-stage security screening system with potential screening errors at each stage, balancing security and congestion in the face of strategic normal and adversary applicants. The authors provide the optimal levels of screening strategies for the approver and the bestresponse application strategies for each type of applicant. This paper provides some novel policy insights which may be useful for security screening practices.

\subsection{Inventory management}

In "An '(s, S)' inventory in a queueing system with batch service facility," Chakravarthy, Maity, and Gupta study a single-server queueing model in which the customers are served in batches of varying size depending on predetermined thresholds as well as available inventory. The authors first analyze their models assuming exponential distribution for the underlying random variables. Then they extend their analysis under more general assumptions on the random variables. The paper "Identification shrinkage in inventory management: an RFID-based solution," by Zhou and Piramuthu, studies the problem of restoring an item's identification information when this has been accidentally lost, typically occurring in retail stores. To address this issue they propose an RFID-enabled tracking/tracing system with a knowledgebased self-adaptive mechanism.

\subsection{Supply chains and logistics}

The paper "On modelling non-linear quantity discounts in a supplier selection problem by mixed linear integer optimization," by Andrade-Pineda, Canca, and Gonzalez-R, addresses a real-world production and transportation problem concerning the processing of biological samples in a large healthcare network. They present a novel approach to solve a multicommodity capacitated network flow problem with concave routing costs, considering outsourcing, overload, and underutilization facility costs. This is followed by paper "An allocation game model with reciprocal behavior and its applications in supply chain pricing decisions," by Zhang, Li, and Gou. The authors propose, as an extension of the standard Stackelberg game, a new allocation game framework that considers reciprocal behavior. They use this framework to investigate the impact of the retailer's reciprocal behavior on pricing decisions in a supply chain pricing problem.

\subsection{Scheduling and project management}

The paper "An integer programming approach to curriculum-based examination timetabling," by Cataldo, Ferrer, Miranda, Rey, and Sauré, studies a particular variant of the examination timetabling problem under two particular features. The authors develop a four-stage integer programming decomposition approach for this problem. Then Durán, Rey, and Wolff, in their paper "Solving the operating room scheduling problem with prioritized lists of patients," develop and compare two integer programming models and two algorithms for scheduling surgical interventions over a defined period that satisfy patient priority criteria. The four mathematical methods were studied under a range of different scenarios using real data from a public hospital in Chile. This is followed by "An MILP model for planning of batch plants 
operating in a campaign-mode," by Fumero, Corsano, and Montagna, who present a mixed integer linear programming model for a production planning problem of multiproduct batch plants. The problem consists of determining the amount of each product to be produced, stored, and sold over the given time horizon; the composition of the production campaign (number of batches and their sizes); the assignment, sequencing, and timing of batches; and the number of repetitions of the campaign, for a given plant with known product recipes. The objective is to maximize the net profit fulfilling the minimum and maximum product demands. In “Fuzzy-probabilistic PERT," Gładysz presents a Program Evaluation and Review Technique (PERT) framework where the individual task time does not follow a known distribution, as is usually assumed. She presents both probabilistic and fuzzy approaches for modeling task duration uncertainty.

\subsection{Forest planning}

Neto, Constantino, Martins, and Pedroso, in their paper "Forest harvest scheduling with clearcut and core area constraints," study forest harvest scheduling problems with constraints on maximum clear-cut size and minimum core habitat area. They present a branch-and-bound procedure designed to find good feasible solutions, in a reasonable time. In each branch of the branch-and-bound tree, a partial solution leads to two children nodes, corresponding to the cases of harvesting or not harvesting a given stand in a given period. Pruning is based on constraint violations or unreachable objective values. Their method was tested with forests ranging from some dozens to more than a thousand stands. This section ends with the paper "The optimal harvesting problem under price uncertainty: the risk averse case," by Pagnoncelli and Piazza, who study the exploitation of a one species, multiple stand forest plantation when timber price is governed by a stochastic process. They propose a stochastic dynamic program model with a weighted mean-risk objective function, using the Conditional Value-at-Risk as their main risk measure. They consider two stochastic processes, namely, geometric Brownian motion and Ornstein-Uhlenbeck, and developed some interesting properties for characterizing optimal solutions.

\subsection{Integer and semi-infinite programming}

The paper "Formulations for the orienteering problem with additional constraints," by Palomo-Martínez, Salazar-Aguilar, and Albornoz, studies a variant of the orienteering problem, taking into account mandatory visits and exclusionary constraints (conflicts among nodes). The authors extend and adapt five mixed integer linear formulations from the traveling salesman problem literature. The main difference among these formulations lies in the way they deal with the subtour elimination constraints. The proposed models are empirically assessed over a large set of instances. In "Improved rapid transit network design model: considering transfer effects," Cadarso and Marín address the rail rapid transit network design problem that consists of locating train alignments and stations and maximizing demand coverage while competing with the current existing network. They present a model for computing tight bounds of the linear relaxation of the problem where transfers are also introduced. The number of transfers within a trip is a decisive attribute for attracting passengers: transferring is annoying and undesirable for passenger. The paper "An asymmetric multi-item auction with quantity discounts applied to Internet service procurement in Buenos Aires public schools," by Bonomo, Catalán, Durán, Epstein, Guajardo, Jawtuschenko, and Marenco, studies a multiitem auction characterized by asymmetric bidders and quantity discounts. The authors report a practical application of this type of auction in the procurement of Internet services in the 
Buenos Aires public school system. They present two integer linear programming models for this problem. This is followed by "A comparative note on the relaxation algorithms for the linear semi-infinite feasibility problem," by Ferrer, Goberna, González-Gutiérrez, and Todorov, who address the linear semi-infinite system feasibility problem (LFP). They propose a new relaxation algorithm with random parameter that is empirically compared with existing work. This new algorithm converges geometrically to a feasible solution under mild conditions. The relaxation algorithms under comparison were implemented using the Extended Cutting Angle Method (ECAM) for solving the global optimization subproblems.

\subsection{Location science}

In "Primal and dual bounds for the vertex $p$-median problem with balance constraints," Díaz and Luna propose a lower bounding scheme based on Lagrangian relaxation and a primal heuristic. This heuristic first provides feasible allocations given a set of medians, then improves the solutions by means of a median exchange procedure. The proposed procedures are empirically assessed in terms of quality and time. In "Incorporating neighborhood reduction for the solution of the planar $p$-median problem," Drezner and Salhi address the $p$-median problem on the plane. The authors present two efficient neighborhood reduction schemes that are embedded into a local search procedure. This local search is also incorporated into one of the most powerful metaheuristics recently developed for this continuous location problem. In "Exact and heuristic approaches for the cycle hub location problem," Contreras, Tanash, and Vidyarthi, study the cycle hub location problem (CHLP), which seeks to locate $p$ hub facilities that are connected by means of a cycle, and to assign non-hub nodes to hubs so as to minimize the total cost of routing flows through the network. This problem is useful in modeling applications in transportation and telecommunications systems, where large setup costs on the links and reliability requirements make cycle topologies a prominent network architecture. The authors present a branch-and-cut algorithm that uses a flow-based formulation and two families of mixed dicut inequalities as a lower bounding procedure at nodes of the enumeration tree. They also introduce a GRASP metaheuristic to obtain initial upper bounds for the exact algorithm and to obtain feasible solutions for large-scale instances of the CHLP. The section ends with the paper "Location-coverage models for preventing attacks on interurban transportation networks," by Auad and Batta, who address the problem of optimally locating patrol units for coping with attacks suffered by interurban road network users. The authors introduce an integer programming model whose objective is to maximize the expected vehicle coverage across the network. The model dynamically locates patrol units through a fixed time horizon, subject to movement and location constraints, considering a probability of not being able to attend to an attack due to a distance factor. They develop some decomposition-based heuristics for this problem.

\subsection{Cutting and packing}

The paper "A heuristic approach to minimize the number of saw cycles in small-scale furniture factories," by Toscano, Rangel, and Yanasse, addresses a two-dimensional cutting stock problem arising in furniture factories. The problem involves the simultaneous optimization of two, usually conflicting, objectives: minimizing the total number of objects and maximizing the cutting machine productivity in terms of the number of objects that are simultaneously cut. The authors propose a heuristic based on variable- and constraint-generation. The main idea is to dynamically add bounds to the frequency of some chosen cutting patterns. A solution is generated in each iteration, reporting a set of non-dominated solutions as final output. They 
tested their approach using real-world data from a small-scale furniture factory. In "Symmetry breaking for generalized disjunctive programming formulation of the strip packing problem," Trespalacios and Grossmann present a new generalized disjunctive programming (GDP) formulation for the strip packing problem. The new formulation helps to break some of the symmetry that arises in this problem. It is further improved for the case in which the heights and lengths of the rectangles are integer numbers. The GDP model can be formulated and solved as a mixed-integer linear programming (MILP) model, using different GDP-to-MILP reformulations. The results show that the MILP reformulations of the new GDP model (and its improvement for rectangles with integer heights and widths) can be solved faster than the previously proposed GDP formulation.

\subsection{Metaheuristics}

Flores-Garza, Salazar-Aguilar, Ngueveu, and Laporte open this section with their paper "The multi-vehicle cumulative covering tour problem." They introduce the multi-vehicle cumulative covering tour problem whose motivation arises from humanitarian logistics. The objective is to determine a set of tours that must be followed by a fleet of vehicles in order to minimize the sum of arrival times (latency) at each visited location. There are three types of locations: mandatory, optional, and unreachable. Each mandatory location must be visited, and optional locations are visited in order to cover the unreachable locations. To guarantee the vehicle autonomy, the duration of each tour should not exceed a given time limit. The authors present a mixed integer linear programming model and a greedy randomized adaptive search procedure for this problem. The paper "New heuristics for the Bicluster Editing Problem," by Filho, Júnior, Cabral, Ochi, and Protti, proposes a set of heuristics using concepts of iterated local search (ILS), VNS, and GRASP, including a constructive heuristic based on analyzing vertex neighborhoods, three local search procedures, and an auxiliary data structure to speed up the local search, for the Bicluster Editing Problem (BEP). The BEP consists of editing a minimum number of edges of an input bipartite graph $\mathrm{G}$ in order to transform it into a vertex-disjoint union of complete bipartite subgraphs. Editing an edge consists of either adding it to the graph or deleting it from the graph. Applications of the BEP include data mining and analysis of gene expression data. In "Optimal location of RFID reader antennas in a three dimensional space," Hervert-Escobar, Smith, Matis, and Vargas-Rosales, propose two optimization models and a GRASP metaheuristic for the problem of determining the number and position of reader antennas in Radio Frequency Identification (RFID) technology. Their approach considers the effect of the orientation of antennas, the type of material to identify, and the interference from obstacles in a three-dimensional warehouse. The solution gives the minimal number of readers along with their positions for the full coverage of the tagged items. Then, de Armas, Cadarso, Juan, and Faulin, in their paper "A multi-start randomized heuristic for real-life crew rostering problems in airlines with work-balancing goals," propose a multi-start randomized heuristic for solving real-world crew rostering problems in airlines. The authors describe constraints, regulations, and rules that have not been considered in the literature so far. Their algorithm aims at balancing the workload distribution among the different crew members. They assess the performance of their heuristic on real-world instances. Sant' Anna, Filomena, Guedes, and Borenstein, in "Index tracking with controlled number of assets using a hybrid heuristic combining genetic algorithm and non-linear programming," address the index tracking problem considering a limited number of assets. The authors develop a hybrid metaheuristic combining the solutions of a nonlinear programming model and a genetic algorithm. They assess their approach on real-world data sets from Ibovespa (the most important Brazilian market index), S\&P 100 (USA), FTSE 
100 (UK), and DAX (Germany). Finally, the section is wrapped up by the paper "Multiobjective variable mesh optimization," by Salgueiro, Toro, Bello, and Falcon, who introduce a new metaheuristic for multi-objective combinatorial optimization (MOCO) based on variable mesh optimization (VMO). Their proposed approach (MOVMO) combines typical concepts from the multiobjective optimization arena, such as Pareto dominance, density estimation, and external archive storage. The metaheuristic also features a crossover operator between local and global optima and dynamic population replacement. They assess their algorithm on a suite of four well-known benchmark function families, and compare it with seven stateof-the-art MOCO methods: NSGA-II, SPEA2, MOCell, AbYSS, SMPSO, MOEA/D, and MOEA/D.DRA.

Acknowledgements We are very grateful to all those who collaborated on this project in one way or another. We would like to thank Professor Endre Boros, the Annals of Operations Research Editor-in-Chief, for his continuing to support the CLAIOs' contributions being published as special issues of this prestigious journal. Special thanks go to Katie D'Agosta, the Annals of Operations Research Publications Manager, for her invaluable help in the editorial process of this volume. She has indeed been involved since the first ANOR issue devoted to CLAIO. Finally, we thank all authors who submitted their work to allow CLAIO and the Annals of Operations Research be the outlet of their fine research work.

Appreciation to Referees Very special thanks go to our referees from institutions around the world whose expertise, time, and effort were translated into prompt and extensive reviews for each submission. Their constructive comments and criticisms contributed significantly to improve the quality of the volume. Unfortunately, we cannot list their names, since the ANOR review process is blind.

\section{Reference}

González-Velarde, J. L., \& Ríos-Mercado, R. Z. (2014). CLAIO Monterrey 2014: A fiesta for the mind and the senses. IFORS News, 8(4), 6-7. 